\title{
Light-induced effects on Spiro-OMeTAD films and hybrid lead halide perovskite solar cells
}

\author{
Rafael S. Sanchez,* Elena Mas-Marza* \\ Institute of Advanced Materials (INAM), Universitat Jaume I, 12071 Castelló, Spain \\ *corresponding authors R.S.S.: $\underline{\text { rasanche@uji.es, E. M.-M.: emas@uji.es }}$
}

4 September 2015

\begin{abstract}
Perovskite (PS) solar cells have emerged as a promising technology for low-cost and efficient photovoltaics. However, the main limiting factors relay on their low long-term stability. The loss of performance of these devices is commonly attributed to degradation of the PS itself. In order to further explore the origins of such limited stability, we have developed UV-VIS spectroscopy studies that clearly demonstrate that PS layers remain unalterable under the working conditions. On the contrary, significant variations in the absorption spectra of the Spiro-OMeTAD layer (Spiro), a commonly used hole transporting material (HTM), indicate that the loss of performance over the irradiation time could be attributed to the degradation of Spiro. UV-VIS measurements evidence a photo-induced oxidation of Spiro, both under air and inert atmosphere. This photo-oxidation is accelerated by the commonly employed additives of the Spiro (LiTFSI and tBP), as well as by the interfacial contact with the electron injecting layer $\left(\mathrm{TiO}_{2}\right)$. Therefore, our results point to the Spiro degradation as the main mechanism that induces the limited functioning of the PS solar cells. Consequently, focusing on the development of alternative HTMs stable under the working conditions is one of the critical issues to be overcome for a suitable progress of PS solar cells and related optoelectronic devices.
\end{abstract}

Keywords: Solar Cell, Perovksite, Spiro-OMeTAD, stability, degradation, photooxidation

\section{Introduction}

In the last few years, hybrid lead halide perovskite (PS) have revolutionized the photovoltaic research at the laboratory scale, mainly due to the relative low-cost techniques required for the PS based solar cells preparation and their outstanding photon-to-current conversion efficiency (PCE); which have reached comparable values to the silicon technology $(\approx 20 \%$ ) [1]. Despite the promising results obtained so far and the encouraging future expectations, several issues and limitations must be overcome in order to make the PS photovoltaics a competitive and convenient technology for the development of high performance large-scale solar panels and their inclusion in the 
market scenario.

PS based solar cells have shown unprecedented phenomena; such as hysteretic behavior [2-5], slow dynamic processes [6], and photo-induced giant dielectric constant [7] [8], which seem to play a significant role on the ultimate performance of the devices and whose exact origin and implications remain still unclear. Additionally, the stability of these devices is considered to form the bottle-neck for a direct application and commercialization of this technology. Although the device stability issue is well-known by the scientific community, only few contributions are focused on the elucidation of the mechanisms that induce the sometimes dramatic drop of the solar cell performance over time. This is a direct consequence of the great variety of external parameters, i.e. illumination, humidity, atmospheric conditions during the preparation, storage and working conditions, which have severe implications on the behavior of this type of devices. In this work, the stability of the materials employed for the preparation of PS based solar cells has been investigated, through a series of optical experiments, when exposed to those conditions required for the characterization and functioning of the complete devices.

\section{Experimental details}

\subsection{Materials preparation}

Solvents and reagents are commercially available and were used as received from commercial suppliers. $\mathrm{CH}_{3} \mathrm{NH}_{3} \mathrm{I}$ was synthesized by reacting $0.273 \mathrm{~mol}$ of $\mathrm{CH}_{3} \mathrm{NH}_{2}$ with $0.223 \mathrm{~mol}$ of aqueous HI. The reactions were carried out in a round bottom flask for $4 \mathrm{~h}$. After this time, the volatiles were removed using a rotary evaporator at $50{ }^{\circ} \mathrm{C}$. The remaining solid was crystallized from ethanol/diethyl ether, filtered, washed with diethyl ether and dried under a vacuum to give $\mathrm{CH}_{3} \mathrm{NH}_{3} \mathrm{I}$ as a white crystalline solid.

\subsection{Films deposition}

Substrate preparation. Fluorine doped tin oxide (FTO) coated glass substrates $(25 \mathrm{x}$ $25 \mathrm{~mm}$, Pilkington TEC15, $\sim 15 \Omega / \mathrm{sq}$ resistance) were cleaned with soap (Hellmanex) and rinsed with milliQ water and ethanol. Then, the sheets were sonicated for 15 minutes in a solution of acetone: isopropanol $(1: 1 \mathrm{v} / \mathrm{v})$, rinsed with ethanol and dried with compressed air. After that, a UV/ozone treatment was performed for 15 minutes.

Compact TiO2 layer $\left(\mathrm{cl}-\mathrm{TiO}_{2}\right)$ deposition. When needed, a $\mathrm{TiO}_{2}$ blocking layer was deposited onto the substrates by spray pyrolysis at $450^{\circ} \mathrm{C}$, using a titanium diisopropoxide bis(acetylacetonate) (75\% in isopropanol, Sigma-Aldrich) solution diluted in ethanol $(1: 39, \mathrm{v} / \mathrm{v})$, with oxygen as carrier gas. After the spraying process the films were kept at $450{ }^{\circ} \mathrm{C}$ for 5 minutes.

Nanostructured $\mathrm{TiO}_{2}$ layer $\left(\mathrm{NS}-\mathrm{TiO}_{2}\right)$ deposition. When needed, a nanostructured $\mathrm{TiO}_{2}$ layer was deposited by spin coating at $4000 \mathrm{rpm}$ during $60 \mathrm{~s}$ using a $\mathrm{TiO}_{2}$ paste 
(Dyesol 18NRT, $20 \mathrm{~nm}$ average particle size) diluted in terpineol (1:3, weight ratio). After drying at $80{ }^{\circ} \mathrm{C}$, the $\mathrm{TiO}_{2}$ layers were heated at $470{ }^{\circ} \mathrm{C}$ for $30 \mathrm{~min}$ and cooled to room temperature.

Perovskite deposition. $100 \mu \mathrm{l}$ of the perovskite precursor solution $(2.64 \mathrm{mmol}$ of $\mathrm{CH}_{3} \mathrm{NH}_{3} \mathrm{I}$ and $0.88 \mathrm{mmol}$ of $\mathrm{PbCl}_{2}$ in $1 \mathrm{~mL}$ of $\mathrm{DMF}$ ) was spin-coated inside the glove box at 2000 r.p.m. for 60 s. After the deposition, the substrate was kept at $100^{\circ} \mathrm{C}$ for 10 min. Next, the substrate wass heated at $100{ }^{\circ} \mathrm{C}$ during 1 hour in an oven under air stream.

Spiro deposition. The Spiro-OMeTAD was depostited by spin coating $100 \mu \mathrm{L}$ of spiro-OMeTAD solution at 4000 r.p.m for $30 \mathrm{~s}$ under air conditions. The spiroOMeTAD solution was prepared by dissolving $72.3 \mathrm{mg}$ of $\left(2,2^{\prime}, 7,7^{\prime}\right.$-tetrakis $\left(\mathrm{N}, \mathrm{N}^{\prime}\right.$-di-pmethoxyphenylamine)-9,9'-spirobifluorene), $28.8 \mu \mathrm{L}$ of 4-tert-butylpyridine and $17.5 \mu \mathrm{L}$ of a stock solution of $520 \mathrm{mg} / \mathrm{mL}$ of lithium bis(trifluoromethylsulphonyl)imide in acetonitrile, in $1 \mathrm{~mL}$ of chlorobenzene.

\subsection{Spiro solutions for UV-VIS measurements}

$20 \mathrm{mg}$ of Spiro were diluted in $2 \mathrm{~mL}$ of toluene. When needed, the additives 4-tertbutylpyridine (tBP, $8 \mu \mathrm{L}$ ) and lithium bis(trifluoromethylsulphonyl)imide (LiTFSI, 4.8 $\mu \mathrm{L}$ of a stock solution of $520 \mathrm{mg} / \mathrm{mL}$ of in acetonitrile) were added. Then, $2 \mu \mathrm{L}$ of the resulting solution were diluted in $3 \mathrm{~mL}$ of acetonitrile to give a $10^{-5} \mathrm{M}$ solution of Spiro, with the corresponding additives when needed.

\section{Results and discussion}

Most of the studies focused on the stability of PS based devices point to the degradation of the perovskite itself as the main mechanism responsible for the loss of the devices performance [9-11]. However, previous works oriented to the characterization of devices using Spiro-OMeTAD as HTM, including solid-state DSSC, demonstrate that Spiro suffers from alterations that have a direct impact on the ultimate performance of the devices $[12,13]$. To further extend these studies to PS based devices, we have developed a series of experiments with the aim of elucidating the origins of the Spiro modifications and their implications on the solar cells performance. The analysis follows the sequence: (1) Demonstration of stability of PS films under illumination. (2) Degradation of Spiro in solution. (3) Degradation of Spiro films.

\subsection{Absorption studies of a PS device and PS film}

In order to study the stability of the materials present in PS solar cells, we exposed under 1 sun (using a solar simulator in air conditions) a complete device without the metal contact (FTO/cl- $\mathrm{TiO}_{2} / \mathrm{PS} / \mathrm{Spiro}$ ) and the evolution of the absorption spectrum over the irradiation time was monitored by means of UV-VIS spectrophotometry (Fig. 1a). It is worth mentioning that the Spiro film was prepared by adding the typical additives (LiTFSI and tBP) employed for increasing its conductivity and doping levels, which is a typical procedure employed to enhance the performance of the devices. 

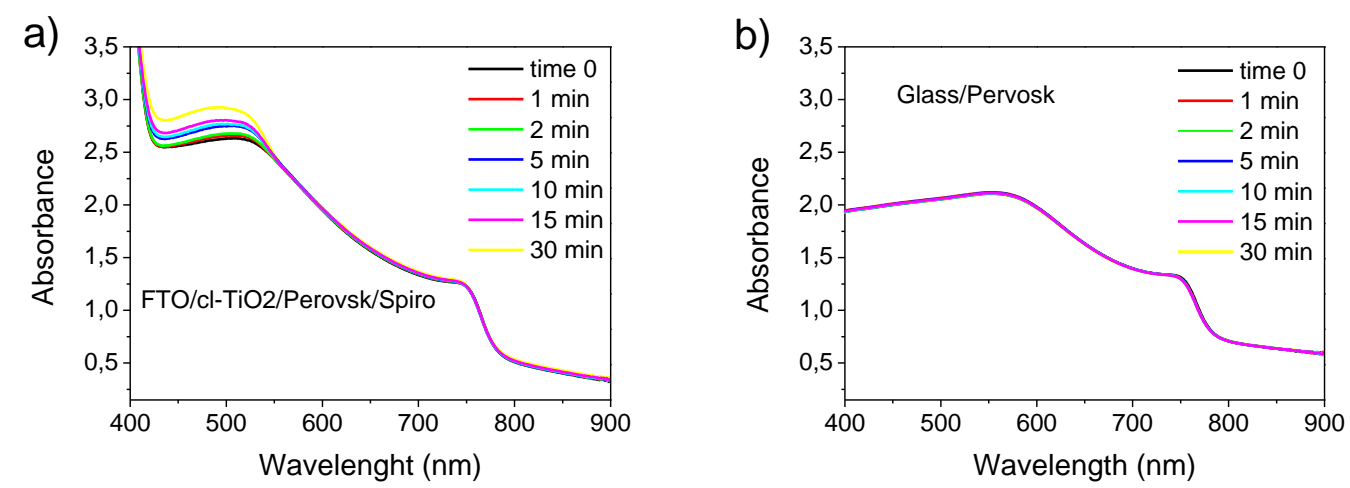

Fig. 1.: a) UV-VIS absorption evolution of a complete device without the top metal contact (FTO/cl$\mathrm{TiO}_{2} / \mathrm{PS} /$ Spiro) and b) of a PS film on glass, at different illumination intervals under the solar simulator.

It is clearly observed in Fig. 1a that for the complete device, the intensity of the absorption signal comprised between 400 and $550 \mathrm{~nm}$ increases over the time of light exposure. However, the absorption bands exclusively ascribed to the PS film do not suffer from significant variations. To further demonstrate that the changes observed cannot be related to a light induced processing of the PS itself, we prepared a PS film onto a glass substrate and the film was light-soaked under the same conditions (Fig. 1b). As it was expected taking into account the previous evidences, the PS absorption signals do not show any significant change along the entire spectral window with the illumination time, thus suggesting that the PS film is stable during the course of the experiment. Therefore, the variations observed in Fig. 1a must be ascribed to a photoinduced modification or degradation of some other material present in the complete device.

Photo-oxidation is one of the main chemical degradation processes when the materials are exposed to intense illumination conditions, especially in the presence of oxidizing agents. In fact, Spiro, which is an organic molecule widely employed as a HTM in solution processed solar cells, is prone to be oxidized from the neutral form (Spiro) to the corresponding radical cation $\left(\mathrm{Spiro}^{+}\right)$due to the withdrawal of an electron, which was confirmed by spectroelectrochemical experiments (see Appendix A, Supporting info. S4). This oxidation process can indeed continue by the sequential loss of a second or even a third electron if the sufficient energy is applied. Additionally, it is broadly accepted in the field of solid state DSSC and PS photovoltaic that a partial oxidation of the Spiro film induces a significant enhancement of the efficiency of the devices due to an increase of the film conductivity and to a doping effect that lowers the Fermi level, thus contributing beneficially to the charge extraction [14]. These advantageous effects are generally induced by adding additives to the Spiro film, i.e. lithium salts, tBP and/or iridium or cobalt based catalysts [15-18].

\subsection{Absorption studies of Spiro solutions}

In order to study the behavior of Spiro under different conditions, we have observed the evolution of a Spiro solution under illumination and dark, in the absence or presence 
of additives (LiTFSI and tBP) and under oxidizing (air) and inert atmosphere (Argon) conditions.
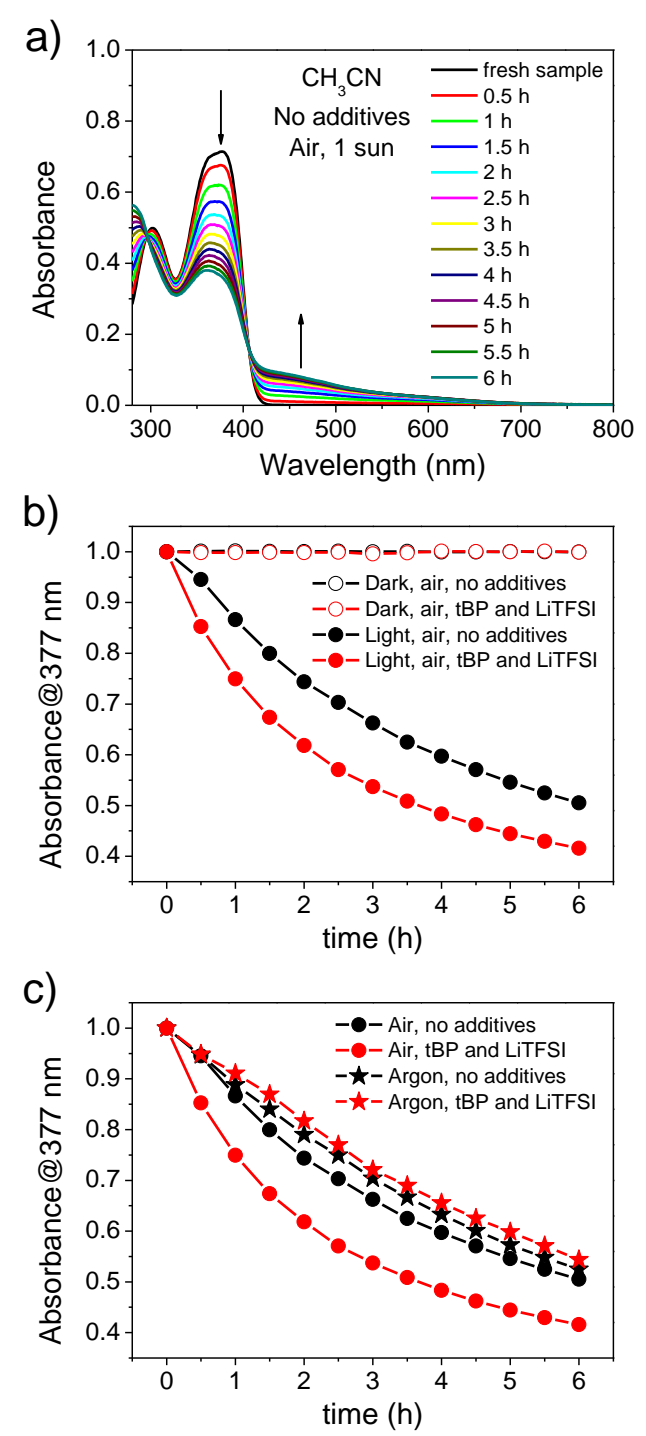

Fig. 2.: a) UV-VIS absorption evolution of a Spiro solution $\left(10^{-5} \mathrm{M}\right)$ in acetonitrile illuminated under 1 sun in air. b) Evolution of the absorbance $\left(\lambda_{\max }=377 \mathrm{~nm}\right)$ in the dark (hollow) and under 1 sun (solid) with the absence (black) or presence (red) of additives in air. c) Evolution of the absorbance $\left(\lambda_{\max }=377\right.$ $\mathrm{nm}$ ) under 1 sun with the absence (black) or presence (red) of additives in air (circle) and in Argon (star).

Fig. 2a shows the UV-VIS absorption spectra of a Spiro solution in acetonitrile at different illumination times in air and without additives. The fresh sample shows an intense absorption signal from 300 to $400 \mathrm{~nm}\left(\lambda_{\max }=377 \mathrm{~nm}\right)$, which is characteristic of the neutral Spiro. When the sample is exposed to light, the absorption band of the neutral species decreases exponentially, while a new signal appears in the visible range comprised between 400 and $700 \mathrm{~nm}$. This behavior has been reported in previous works and it was ascribed to the oxidation of the neutral Spiro towards the formation of the radical cation Spiro ${ }^{+}$. Most of the works reporting on these observations attribute the 
formation of the radical cation to the addition of the additives LiTFSI and/or tBP [15, 19], or even to an oxygen-induced effect [12]. Nevertheless, up to our knowledge, only few manuscripts report on the effect of light on the oxidation of the Spiro [13].

Fig. $2 b$ shows the evolution of the absorption band of the neutral Spiro $\left(\lambda_{\max }=377\right.$ $\mathrm{nm}$ ) in the dark and under 1 sun, with or without additives in air atmosphere. Here, we clearly demonstrate that the addition of additives even in air conditions does not induce any observable oxidation of the Spiro molecule when the sample is stored in the dark, at least in the course of our experiments. However, when the sample is exposed to light, the formation of Spiro ${ }^{+}$is promoted, thus observing a decrease of the absorption band intensity of the neutral state. In any case, the presence of additives (LiTFSI and tBP) accelerates the kinetics of the oxidation reaction, probably due to the stabilization of the Spiro radical cation. Fig. 2c shows the evolution of the same absorption band under inert atmosphere (Argon), and as it can be seen, the oxidation also occurs, although this process is significantly slower. Therefore, from these results it can be concluded that the oxidation of Spiro is exclusively a photo-induced phenomenon that is accelerated by the presence of oxidizing agents, e.g. oxygen, and/or additives. It is worth remarking this statement, since the working conditions of a solar cell involve relatively intense illumination levels, thus implying a continuous evolution that might have a deep impact on the performance of devices over the time.

\subsection{Absorption studies of Spiro films}

In order to obtain information on the behavior of Spiro in conditions comparable to a solid state solar cell, we designed a series of experiments on films.

a)

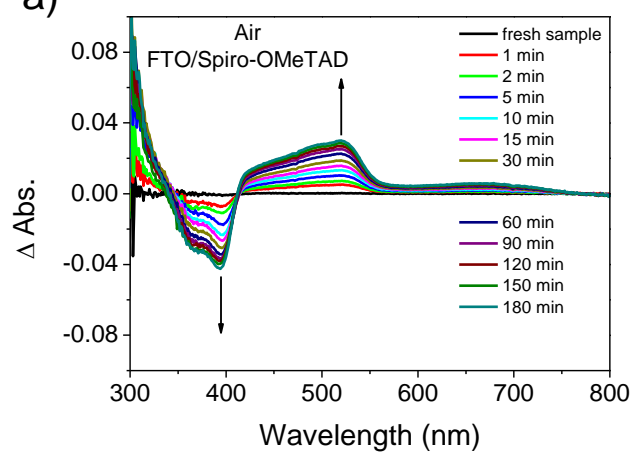

b)

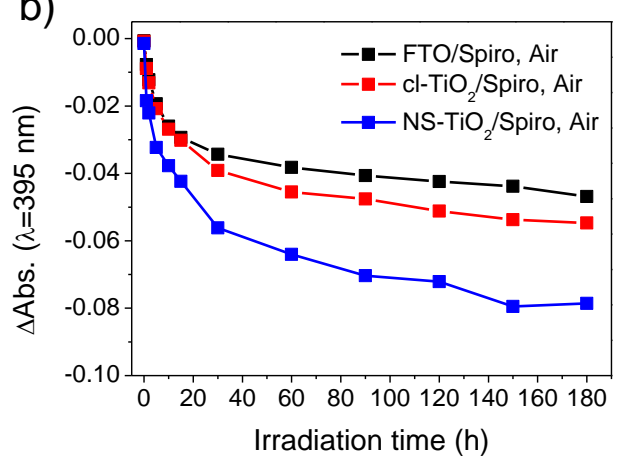

Fig. 3.: a) Evolution of the UV-VIS absorption signals of a Spiro film deposited by spin-coating on a FTO substrate. b) Evolution of the absorbance $\left(\lambda_{\max }=395 \mathrm{~nm}\right)$ under 1 sun of a Spiro film deposited on FTO (black), cl- $\mathrm{TiO}_{2}$ (red) and $\mathrm{NS}^{-\mathrm{TiO}_{2}}$ (blue) in air, respectively.

Fig. 3a shows the evolution of the delta absorbance ( $\triangle \mathrm{Abs}$.) of a Spiro film deposited onto a FTO substrate with the illumination time (1 sun, in air). As it can be observed, the behavior of the Spiro film resembles the trend observed in solution. In this case, the $\lambda_{\max }$ of the absorption band of the neutral specie is red-shifted and centered at $395 \mathrm{~nm}$, which is consistent with the enhanced intermolecular interactions due to the formation of a film. Since Fig. 3a plots the $\triangle \mathrm{Abs}$., the neutral Spiro absorption band appears in the 
negative region as long as the oxidation takes place. However, those bands ascribed to the Spiro ${ }^{+}$species are positive and are observable over the entire visible range (400-750 $\mathrm{nm})$. This experiment demonstrates that the oxidation of the Spiro film is significantly observed even at short illumination intervals ( 1 minute). Fig. $2 b$ shows the evolution of the $\triangle \mathrm{Abs}$. at $395 \mathrm{~nm}$ over the illumination time of the Spiro films deposited on FTO, compact $\mathrm{TiO}_{2}\left(\mathrm{cl}-\mathrm{TiO}_{2}\right)$ and nanostructured $\mathrm{TiO}_{2}\left(\mathrm{NS}-\mathrm{TiO}_{2}\right)$, respectively. As it can be clearly observed, the photo-oxidation kinetics of the Spiro molecules is accelerated by the $\mathrm{TiO}_{2}$ [12], which is especially notable for the $\mathrm{NS}-\mathrm{TiO}_{2}$, probably due to a larger surface contact effect. These evidences correlate with the importance of preparing PS films with a good surface coverage, i.e. pin-hole free films, for producing high performance solar cells, since direct contact between the Spiro and $\mathrm{TiO}_{2}$ films might not only induce an enhancement of the charge recombination pathways but also a faster degradation of the Spiro film.

The extinction coefficients of the Spiro and Spiro $^{+}$species were determined in previous works, being $75000 \mathrm{M}^{-1} \cdot \mathrm{cm}^{-1}$ for the neutral Spiro at $385 \mathrm{~nm}$ [20], $51000 \mathrm{M}^{-}$ ${ }^{1} \cdot \mathrm{cm}^{-1}$ for the Spiro ${ }^{+}$at $385 \mathrm{~nm} \mathrm{[19]} \mathrm{and} 33000 \mathrm{M}^{-1} \cdot \mathrm{cm}^{-1}$ for the $\mathrm{Spiro}^{+}$at $500 \mathrm{~nm} \mathrm{[21].}$ By using these values and equation 1, we have roughly estimated the molar ratio Spiro $^{+} /$Spiro at the different illumination intervals for the FTO/Spiro film (Fig. 4).

$$
\frac{\left[\text { Spiro }^{+}\right]}{[\text {Spiro }]}=\frac{\text { Abs }^{\lambda=500 \mathrm{~nm}} \cdot \varepsilon_{\text {Spiro }}^{\lambda=385 \mathrm{~nm}}}{\left(\text { Abs }^{\lambda=385 \mathrm{~nm}} \cdot \varepsilon_{\text {Spirot }}^{\lambda=500 \mathrm{~nm}}\right)-\left(\text { Abs }^{\lambda=500 \mathrm{~nm}} \cdot \varepsilon_{\text {Spiro+ }}^{\lambda=385 \mathrm{~nm}}\right)}
$$

eq. 1

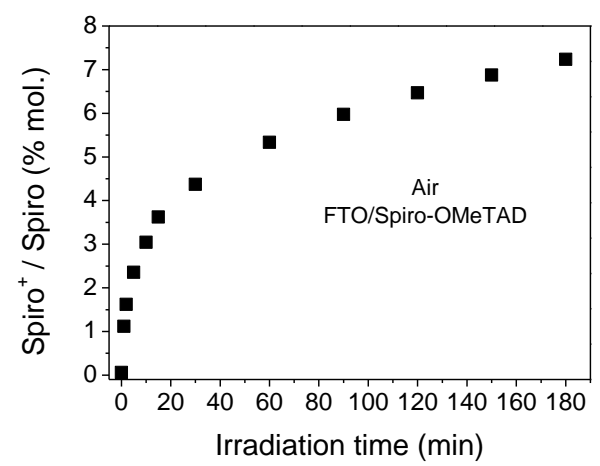

Fig. 4.: estimation of the Spiro $^{+} /$Spiro molar ratio of a FTO/Spiro film under 1 sun at different illumination times.

It is widely accepted that doping the Spiro film, by adding lithium salts, metal catalysts or by inducing a partial oxidation, results in a downward shift of the Fermi level $\left(E_{f}\right)$, which enhances the conductivity of the film $[14,22]$ and allows adjusting the energy levels for an optimum charge extraction [19, 23]. Some precedent works found in the literature emphasize the crucial role of controlling the doping level of the Spiro films with the aim of enhancing the overall performance of the devices [14, 19]. In fact, it has been demonstrated that low doping levels of Spiro layer contribute beneficially to 
the device functioning in solid-state DSSC; however, after reaching a threshold the performance drops mainly due to a decrease of the $J_{s c}$ [13].

Fig. 4 shows the evolution of the Spiro ${ }^{+} /$Spiro molar ratio of a FTO/Spiro film at different illumination intervals, achieving significant values of molar ratio $(\approx 10 \%$ in 3 hours). Here we demonstrate that the oxidation of the Spiro film evolves exponentially with the illumination time. Therefore, it is expected that a complete device will suffer a similar photo-induced evolution in the working conditions, thus making difficult a precise control of the Spiro doping level. In fact, a related phenomenon has been recently reported for PS based LEDs, where an initial partial oxidation of the Spiro film provokes an enhancement of the device performance; however after reaching an oxidation threshold, the performance starts dropping dramatically [24]. Therefore, such oxidation has direct consequences on the device functioning and stability. Even though this is out of the scope of this contribution, the continuous alterations of the HTM film might have some implications on the hysteretic behavior of $J / V$ curves and/or slow dynamic processes observed in PS based solar cells.

\section{Conclusions}

In summary, we have demonstrated that Spiro-OMeTAD suffers from a photoinduced oxidation not only in solution but also on a film. This oxidation is facilitated in an oxidizing environment (air) but being also significant under inert atmosphere (Argon). In addition, the use of LiTFSI and tBP additives accelerates this photooxidation process. Our results also highlight the essential role of improving the PS deposition methodologies with the aim of avoiding a direct contact between the Spiro and $\mathrm{TiO}_{2}$ films to deactivate an additional photo-oxidation pathway and subsequent loss of performance of the devices. Therefore, we conclude that the photo-degradation of Spiro seems to be one of the limiting factors for achieving long-term stable PS solar cell. Consequently, focusing on the preparation of alternative materials with good charge selective properties as HTM and enhanced photo-stability seems to be a crucial limitation to be overcome for the preparation of high performance and long-term stability solar cells.

\section{Acknowledgement}

The work was supported by MINECO of Spain under project ENE2014-56237-C4-3R. E.M.-M thanks the Ramón y Cajal program from MINECO of Spain and R.S.S. thanks the FP7 European project ALLOXIDE (309018). The authors also thank Prof. Juan Bisquert for useful discussions and support.

\section{References}

[1] http://www.nrel.gov/ncpv/images/efficiency_chart.jpg.

[2] H.J. Snaith, A. Abate, J.M. Ball, G.E. Eperon, T. Leijtens, N.K. Noel, S.D. Stranks, J.T.-W. Wang, K. Wojciechowski, W. Zhang, Anomalous Hysteresis in Perovskite 
Solar Cells, The Journal of Physical Chemistry Letters, 5 (2014) 1511-1515.

[3] E.L. Unger, E.T. Hoke, C.D. Bailie, W.H. Nguyen, A.R. Bowring, T. Heumueller, M.G. Christoforo, M.D. McGehee, Hysteresis and transient behavior in currentvoltage measurements of hybrid-perovskite absorber solar cells, Energy \& Environmental Science, 7 (2014) 3690-3698.

[4] N.J. Jeon, J.H. Noh, Y.C. Kim, W.S. Yang, S. Ryu, S. Il Seol, Solvent engineering for high-performance inorganic-organic hybrid perovskite solar cells, Nature Materials, 13 (2014) 897-903.

[5] L.K. Ono, S.R. Raga, S.H. Wang, Y. Kato, Y.B. Qi, Temperature-dependent hysteresis effects in perovskite-based solar cells, Journal of Materials Chemistry A, 3 (2015) 9074-9080.

[6] R.S. Sanchez, V. Gonzalez-Pedro, J.-W. Lee, N.-G. Park, Y.S. Kang, I. Mora-Sero, J. Bisquert, Slow Dynamic Processes in Lead Halide Perovskite Solar Cells. Characteristic Times and Hysteresis, The Journal of Physical Chemistry Letters, 5 (2014) 2357-2363.

[7] E.J. Juarez-Perez, R.S. Sanchez, L. Badia, G. Garcia-Belmonte, Y.S. Kang, I. MoraSero, J. Bisquert, Photoinduced Giant Dielectric Constant in Lead Halide Perovskite Solar Cells, The Journal of Physical Chemistry Letters, 5 (2014) 2390-2394.

[8] D.P. Almond, C.R. Bowen, An Explanation of the Photoinduced Giant Dielectric Constant of Lead Halide Perovskite Solar Cells, The Journal of Physical Chemistry Letters, 6 (2015) 1736-1740.

[9] G. Niu, X. Guo, L. Wang, Review of recent progress in chemical stability of perovskite solar cells, Journal of Materials Chemistry A, 3 (2015) 8970-8980.

[10] J. Yang, B.D. Siempelkamp, D. Liu, T.L. Kelly, Investigation of CH3NH3PbI3 Degradation Rates and Mechanisms in Controlled Humidity Environments Using in Situ Techniques, Acs Nano, 9 (2015) 1955-1963.

[11] Y. Han, S. Meyer, Y. Dkhissi, K. Weber, J.M. Pringle, U. Bach, L. Spiccia, Y.-B. Cheng, Degradation observations of encapsulated planar $\mathrm{CH} 3 \mathrm{NH} 3 \mathrm{PbI} 3$ perovskite solar cells at high temperatures and humidity, Journal of Materials Chemistry A, 3 (2015) 8139-8147.

[12] U.B. Cappel, T. Daeneke, U. Bach, Oxygen-induced doping of spiro-MeOTAD in solid-state dye-sensitized solar cells and its impact on device performance, Nano letters, 12 (2012) 4925-4931.

[13] H. Wang, M. Xu, G. Liu, X. Li, P. Xiang, Z. Ku, Y. Rong, L. Liu, M. Hu, Y. Yang, Effect of photo-doping on performance for solid-state dye-sensitized solar cell based on 2, 2' 7, 7'-tetrakis-(N, N-di-p-methoxyphenyl-amine)-9, 9'-spirobifluorene and carbon counter electrode, Electrochimica Acta, 99 (2013) 238-241. 
[14] W.H. Nguyen, C.D. Bailie, E.L. Unger, M.D. McGehee, Enhancing the holeconductivity of spiro-OMeTAD without oxygen or lithium salts by using spiro (TFSI) 2 in perovskite and dye-sensitized solar cells, Journal of the American Chemical Society, 136 (2014) 10996-11001.

[15] A. Abate, T. Leijtens, S. Pathak, J. Teuscher, R. Avolio, M.E. Errico, J. Kirkpatrik, J.M. Ball, P. Docampo, I. McPherson, Lithium salts as "redox active" p-type dopants for organic semiconductors and their impact in solid-state dye-sensitized solar cells, Physical Chemistry Chemical Physics, 15 (2013) 2572-2579.

[16] L. Badia, E. Mas-Marza, R.S. Sanchez, E.M. Barea, J. Bisquert, I. Mora-Sero, New iridium complex as additive to the spiro-OMeTAD in perovskite solar cells with enhanced stability, Apl Materials, 2 (2014).

[17] J.H. Noh, N.J. Jeon, Y.C. Choi, M.K. Nazeeruddin, M. Gratzel, S.I. Seok, Nanostructured $\mathrm{TiO} 2 / \mathrm{CH} 3 \mathrm{NH} 3 \mathrm{PbI} 3$ heterojunction solar cells employing spiroOMeTAD/Co-complex as hole-transporting material, Journal of Materials Chemistry A, 1 (2013) 11842-11847.

[18] W.H. Howie, J.E. Harris, J.R. Jennings, L.M. Peter, Solid-state dye-sensitized solar cells based on spiro-MeOTAD, Solar Energy Materials and Solar Cells, 91 (2007) 424-426.

[19] R. Schölin, M.H. Karlsson, S.K. Eriksson, H. Siegbahn, E.M. Johansson, H. Rensmo, Energy level shifts in spiro-OMeTAD molecular thin films when adding LiTFSI, The Journal of Physical Chemistry C, 116 (2012) 26300-26305.

[20] I. Ding, N. Tétreault, J. Brillet, B.E. Hardin, E.H. Smith, S.J. Rosenthal, F. Sauvage, M. Grätzel, M.D. McGehee, Pore-Filling of Spiro-OMeTAD in Solid-State Dye Sensitized Solar Cells: Quantification, Mechanism, and Consequences for Device Performance, Advanced Functional Materials, 19 (2009) 2431-2436.

[21] U.B. Cappel, E.A. Gibson, A. Hagfeldt, G. Boschloo, Dye regeneration by spiroMeOTAD in solid state dye-sensitized solar cells studied by photoinduced absorption spectroscopy and spectroelectrochemistry, The Journal of Physical Chemistry C, 113 (2009) 6275-6281.

[22] H.J. Snaith, M. Grätzel, Enhanced charge mobility in a molecular hole transporter via addition of redox inactive ionic dopant: Implication to dye-sensitized solar cells, Applied physics letters, 89 (2006) 2114.

[23] Z. Hawash, L.K. Ono, S.R. Raga, M.V. Lee, Y. Qi, Air-Exposure Induced Dopant Redistribution and Energy Level Shifts in Spin-Coated Spiro-MeOTAD Films, Chemistry of Materials, 27 (2015) 562-569.

[24] O.A. Jaramillo-Quintero, R.S. Sanchez, M. Rincon, I. Mora-Sero, Bright VisibleInfrared Light Emitting Diodes Based on Hybrid Halide Perovskite with Spiro- 
OMeTAD as a Hole-Injecting Layer, The Journal of Physical Chemistry Letters, 6 (2015) 1883-1890. 
APPENDIX A. SUPPORTING INFORMATION

\section{Light-induced effects on Spiro-OMeTAD films and hybrid lead halide perovskite solar cells}

\section{Rafael S. Sanchez,* Elena Mas-Marza*}

Institute of Advanced Materials (INAM), Universitat Jaume I, 12071 Castelló, Spain

*corresponding authorsR.S.S.: rasanche@uji.es, E. M.-M.: emas@uji.es

\section{Instrument description}

UV/Vis absorption spectra were recorded on a Varian Cary 300 BIO spectrophotometer in $\mathrm{CH}_{3} \mathrm{CN}$ solution or on films under air or inert conditions (Argon).

\section{Optical characterization}

2.1 Evolution of the Spiro-OMeTAD absorption spectra in solution

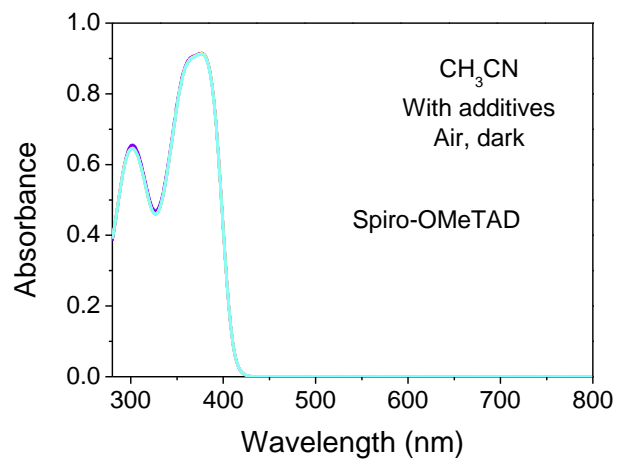

S1.: UV-VIS absorption evolution of a Spiro solution $\left(10^{-5} \mathrm{M}\right)$ in acetonitrile in dark during 6 hours under air with additives (LiTFSI and tBP).

2.2 Evolution of the Spiro-OMeTAD absorption spectra on film

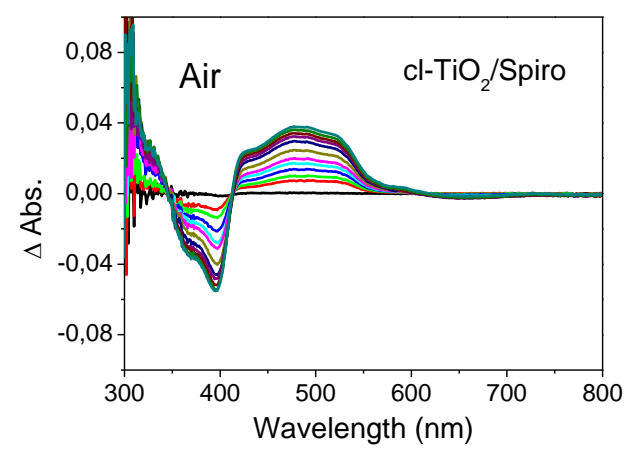

S2.: Evolution of the UV-VIS absorption signals of a Spiro film deposited by spin-coating on a cl- $\mathrm{TiO}_{2}$ layer. 


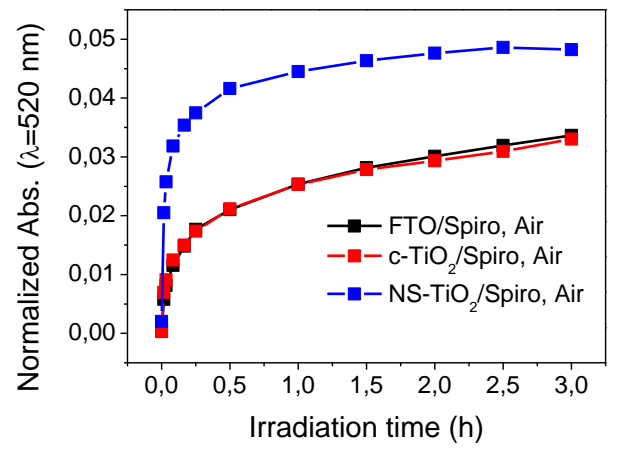

S3.: Evolution of the absorbance $\left(\lambda_{\max }=520 \mathrm{~nm}\right)$ under 1 sun of a Spiro film deposited on FTO (black), cl- $-\mathrm{TiO}_{2}$ (red) and $\mathrm{NS}^{-\mathrm{TiO}_{2}}$ (blue) in air, respectively.

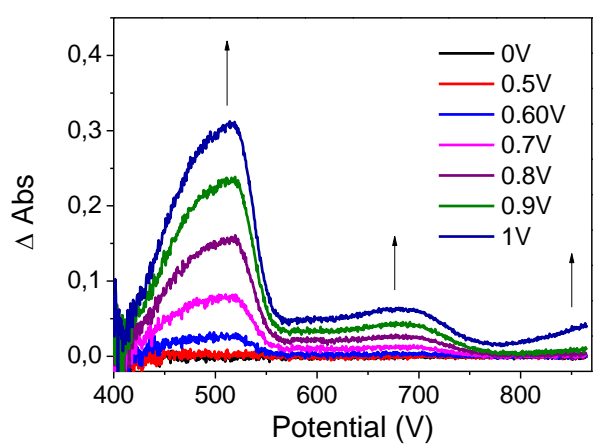

S4.: Spectroelectrochemistry of a Spiro film deposited on a FTO substrate in Propylene carbonate using $\mathrm{TBAPF}_{6}(0.1 \mathrm{M})$ as supporting electrolyte and measured at a scan-rate of $100 \mathrm{mV} \cdot \mathrm{s}^{-1}$. The potential values were measured using an $\mathrm{Ag} / \mathrm{AgCl}$ reference electrode and a Pt counter electrode. 